\title{
Pseudokaposi's sarcoma: a diagnostic dilemma
}

\section{Nagaratna Chatnallikar, Leelavthy Budamakuntala, Shilpa Kapanigowda}

\author{
Department of Dermatology Bangalore Medical College and Research Institute. Kalasipalyam. Bangalore. Karnataka. India
}

Corresponding author: Dr. Nagaratna Chatnallikar, E-mail: nagratna.c@gmail.com

\begin{abstract}
Acroangiodermatitis of mali also called as pseudokaposi s sarcoma is a very rare, benign condition which clinically presents as purple-colored patches, plaques or nodules, mostly on the extensor surfaces of lower extremities in patients with chronic venous insufficiency and arteriovenous malformations. It resembles clinically with conditions like Kaposi's sarcoma and hence requires histopathological examination for its diagnosis. We report one such case of acroangiodermatitis. 45 year old male presented with multiple violaceus colored plaque with ulceration on the left extensor aspect of the leg, with associated venous insufficiency of same limb. On histopathology it showed proliferating small vessels associated with clusters of spindle cells showing numerous extravasated red blood corpuscles (RBCs), scattered plump to ovalendothelial cells with dark nuclei in the centre. Patient was treated with oral erythromycin and lesions improved in 3 weeks.
\end{abstract}

Key words: Venous insufficiency; Pseudokaposi s sarcoma; Immunohistochemistry; Klippel-Trenaunay syndrome

\section{INTRODUCTION}

Acroangiodermatitis is a reactive angiodysplasia of cutaneous blood vessels associated with venous insufficiency or with vascular anomalies such as Klippel-Trenaunay syndrome or stump dermatosis in amputees. Exaggerated stasis dermatitis begins as violaceous macules and patches that gradually develop into papule, nodules, or indurated plaques often bilateral, and usually located on the lower extremities with edema. Rarely it can present with ulceration also. Although benign, it may mimic malignant conditions like Kaposi's sarcoma and therefore requires histopathological examination for its diagnosis and differentiation.

Here, we report one such case presenting with nodulo-ulcerative lesions, with histological features of acroangiodermatitis, emphasizing the importance of histopathology and immunohistochemistry in differentiating this from similarly presenting condition.

\section{CASE REPORT}

A 45 year old male presented with multiple ulcerated lesions over the left leg with associated discharge since 6 months. It started spontaneously, gradually spreading to involve other areas of left leg. No history of trauma, asoociated pain. The discharge was minimal, non foul smelling. No history of any grain like discharge. Patient is a known case of alcoholic liver disease. No other systemic illness or major surgery in the past.

\section{On examination}

1. Single deep ulcer measuring about $1 * 1 \mathrm{~cm}$, well defined margins, sloping edges, base formed by granulation tissue, non tender with minimal purulent discharge present over the medial aspect of left leg.

2. Single subcutaneous mass measuring about $5 * 3 \mathrm{~cm}$ over the medial malleolus with overlying small ulcer covered with healthy granulation tissue (Fig. 1).

3. Multiple hyperpigmented plaque of varying size with few showing surrounding violaceous hue over the extensor aspect of left leg.

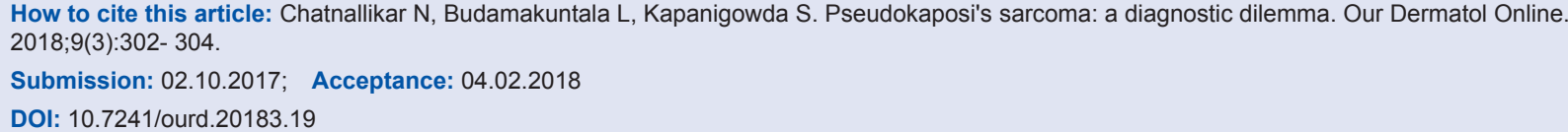


4. Non pitting odema of the left leg till the mid calf was present. Superficial varicosites were visible. Right leg was normal.

Based on this a differential diagnosis of mycetoma, Kaposi s sarcoma, pseudokaposi's sarcoma, bartenlosis was considered.

On investigating, all routine haematological and biochemical investigations were normal, ELISA for HIV was negative. $\mathrm{KOH}$ was negative, gram stain showed few pus cells, gram positive cocci in singles and pairs, occasional gram negative bacilli were present. AFB was negative.

On histopathological examination epidermis showed psoriasiform acanthosis, papillomatosis, increased vascular proloiferation with cluster of spindle cells showing numerous extravasated RBCs (Fig. 2). Immunohistochemistry was positive for CD 31 and factor VII, confined to vessels and endothelium.

Venous doppler of left limb showed incompetent sapheno femoral junction.

Final diagnosis of pseudokaposi s sarcoma was made.

Patient was treated with oral erythromycin $500 \mathrm{mg}$ QID and compression stockings was advised (Fig. 3).

Prior to the study, patient gave written consent to the examination and biopsy after having been informed about the procedure.

\section{DISCUSSION}

Acroangiodermatitis (Synonyms: pseudo-Kaposi's sarcoma, acroangiodermatitis of Mali-Kuiper, gravitational purpura, stasis purpura) was first coined by Mali in 1965 [1]. It is a proliferation of pre-existing vasculature seen in chronic venous insufficiency, arteriovenous malformation, or acquired iatrogenic arteriovenous (AV) fistula. A variety of vascular conditions giving rise to this venous pathology include Klippel-Trenaunay syndrome, stump dermatosis in amputees, paralyzed limb, and intravenous drug abuse. It is rarely reported in hereditary coagulation defects (carrier of the thrombophilic 20210A mutation in the prothrombin gene and homozygous activated protein $\mathrm{C}$ resistance) $[2,3]$. Most of the cases of acroangiodermatitis have been associated with some signs of venous insufficiency however
Barbar et al. reported no venous insufficiency in 9 of their 10 case of acroangiodermatitis [4] and few cases of spontaneous presentation have also been reported [5].

There are various variants of acroangiodermatitis:

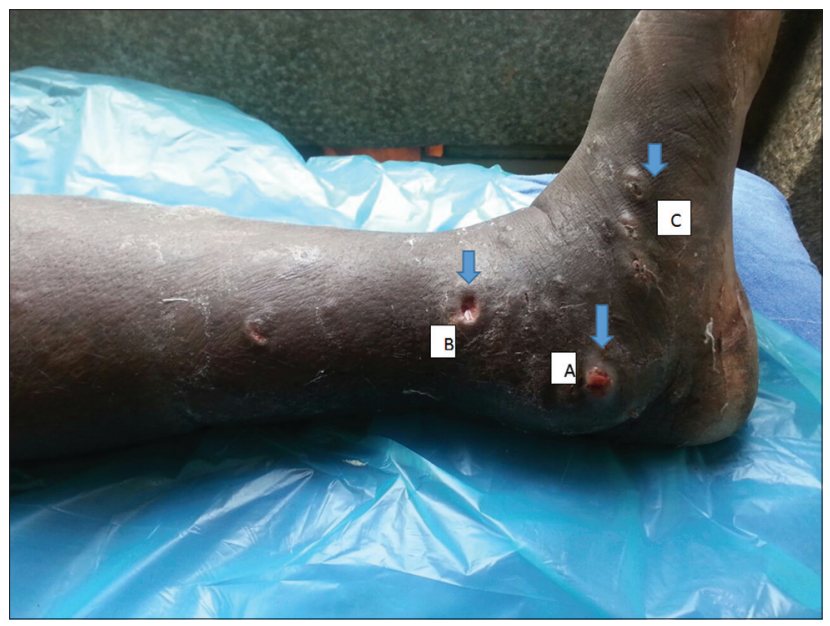

Figure 1: Single subcutaneous mass over the medial malleolus with overlying small ulcer covered with healthy granulation tissue $(A)$, single deep ulcer with minimal purulent discharge present over the medial aspect of left leg (B) and multiple hyperpigmented plaques (C).

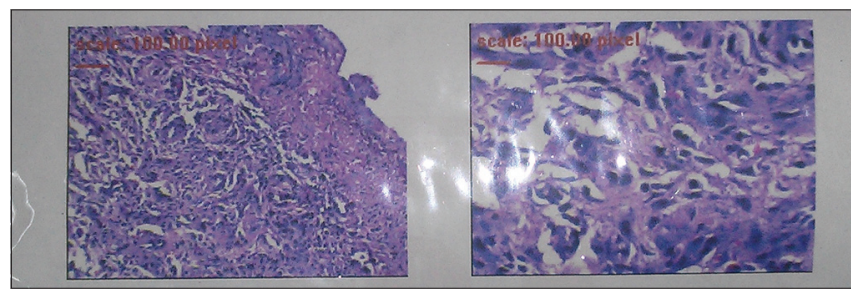

Figure 2: Histopathology showing psoriasiform acanthosis, papillomatosis, increased vascular proloiferation with cluster of spindle cells showing numerous extravasated RBCs.

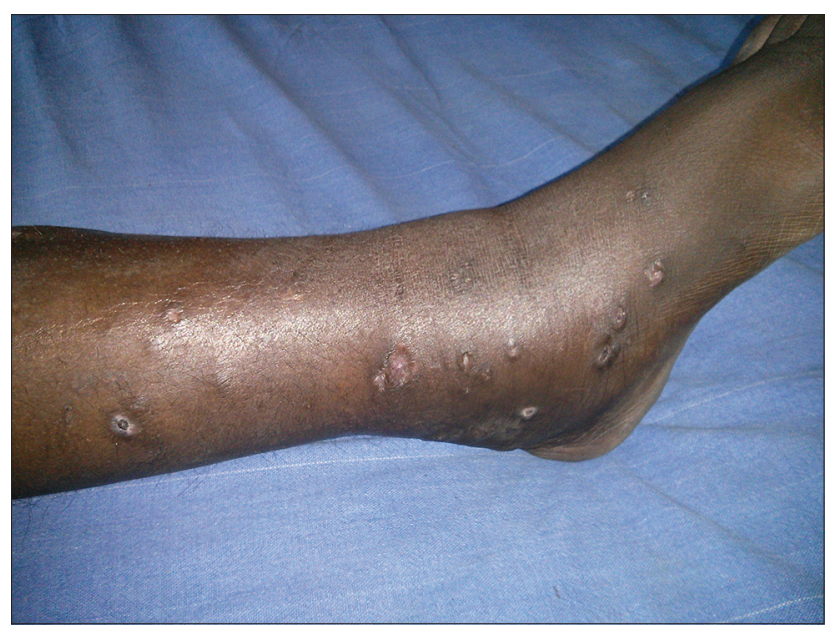

Figure 3: Lesions improved after treatment with oral doxycycline and compression stockings. 
- Stewart-Bluefarb syndrome is a congenital arteriovenous malformation of the lower leg with multiple arteriovenous shunts. It begins early in life unilaterally over lower extremities with painful purple papules and macules, which may ulcerate. The affected limb may show increased warmth with varicose veins and a palpable thrill can be felt [1]

- Mali type is an exaggerated stasis dermatitis seen in elderly patients, usually bilateral with chronic venous insufficiency on dorsum of feet, hallux, and second toe or on medial aspect of lower legs. The lesions begin as violaceous macules and patches that develop slowly into soft, non tender, red to purple papules and nodules or indurated plaques [1].

- Dermite ocre of Favre (gravity purpura): Venous varicosities with first pregnancy [6].

- Angiodermatitis occurring after placement of the arteriovenous shunt for hemodialysis in chronic renal failure [5].

- Although precise etiology is unknown, it is postulated that severe chronic venous stasis with insufficiency of the calf muscle pump elevates the capillary pressure and leads to chronic tissue hypoxia from chronic edema which induces neovascularisation and fibroblast proliferation.

- Chronic pressure changes with the vessel proliferation in the upper and mid-dermis andextravasation of red blood cells produce a combination of purplish papules and plaques on the edematous skin [7].

- Severe chronic venous stasis with insufficiency of the calf muscle pump elevates the capillary pressure and leads to chronic tissue hypoxia from chronic edema. and this in turn to neovascularisation and fibroblast proliferation.

- Clinically it presents clinically as multiple papules, nodules and plaques, sharply defined reddishbrown, sometimes in bizarre configurations, generally localized on the dorsal aspect of the foot and the lower aspect of the shin, usually associated with edema.

- Histopathological examination shows proliferation of endothelial cells, newly- formed vessels with thick walls, often in a lobular pattern and surrounded by pericytes in the dermis.

- Extravasation of red blood cells, hemosiderin pigment deposition, superficial perivascular infiltrate of lymphocytes, histiocytes and occasional plasma cells are also found and may resemble Kaposi's sarcoma.
- Immunohistochemical staining with Cluster of differentiation (CD34) antiserum helps to distinguish between acroangiodermatitis and Kaposi's sarcoma, because in the former, an absence of perivascular CD34 is noted, unlike in the latter (CD34 staining on the endothelial cells as well as the perivascular spindle cells)

- Various medical modalities of therapy have been tried with favorable results, but options are limited.

- Oral erythromycin 500 mg four times a day or dapsone $50 \mathrm{mg}$ twice a day for 3 months in combination with compression therapy has been tried with good results [3].

- Topical therapy with local corticosteroid preparations is often useful [6].

\section{CONCLUSION}

This case report is to emphasize the importance of histopathology and immunohistochemistry to differentiate this benign condition from other similar presentation of malignant conditions.

\section{CONSENT}

The examination of the patient was conducted according to the Declaration of Helsinki principles.

\section{REFERENCES}

1. Agrawal S, Rizal A, Agrawal C, Agrawal A. Pseudo-Kaposi's sarcoma (Bluefarb-Stewart type). Int J Dermatol. 2005;44:136-8.

2. Rongioletti F, Rebora A. Cutaneous reactive angiomatoses: Patterns and classification of reactive vascular proliferation. J Am Acad Dermatol. 2003;49:887-96.

3. Coban I, Kokenek-Unal TD, Alper M. Spontaneous Acroangiodermatitis. Indian J Dermatol. 2015;60:268-71.

4. Rao B, Unis M, Poulos E. Acroangiodermatitis: A study of ten cases. Int J Dermatol. 1994;33:179-83

5. Hung NA, Strack M, Van Rij A, North CJ, Blennerhassett JB. Spontaneous acroangiodermatitis in a young woman. Dermatol Online J. 2004;10:8.

6. Ghia DH, Nayak CS, Madke BS, Gadkari RP. Stewart-Bluefarb acroangiodermatitis in a case of Parkes-Weber syndrome. Indian J Dermatol 2014;59:406-8.

7. Singh SK, Manchanda K. Acroangiodermatitis (Pseudo-Kaposi sarcoma). Indian Dermatol Online J. 2014;5:323-5.

Copyright by Nagaratna Chatnallikar, et al. This is an open-access article distributed under the terms of the Creative Commons Attribution License, which permits unrestricted use, distribution, and reproduction in any medium, provided the original author and source are credited.

Source of Support: Nil, Conflict of Interest: None declared. 